\title{
Study on plating parameters for preparing Ni-TiN nanocoatings by using Artificial Neural Networks
}

\author{
Fafeng Xia ${ }^{1, a}$, Wanchun Jia ${ }^{1, b}$, Chunyang Ma ${ }^{1, ~ c *}$ \\ ${ }^{1}$ School of Mechanical Science and Engineering, Northeast Petroleum University, \\ Daqing 163318, China \\ axiaff@126.com; bwanchun331@126.com; chunyangandma@163.com
}

Keywords: Plating parameter; Ni-TiN nanocoating; Forecast; Artificial Neural Networks

\begin{abstract}
Ni-TiN nanocoatings were successfully synthesized on the surface of 45 steel substrates by using ultrasonic-electrodeposition method. The plating parameters for preparing Ni-TiN nanocoatings were optimized by using an artificial neural network (ANN) basing on the orthogonal test. And the morphologies of the coatings were investigated via scanning electron microscope (SEM). The results indicated that the optimal synthetic conditions were determined as follows: the concentration of TiN nanoparticles $7.5 \mathrm{~g} / \mathrm{L}$, the current density $5 \mathrm{~A} / \mathrm{dm}^{2}$, the duty cycle $3: 2$, the concentration of surfactants $80 \mathrm{mg} / \mathrm{L}$ and the ultrasonic power $250 \mathrm{~W}$. The Ni-TiN nanocoating prepared under the optimal synthetic conditions had a compact and fine microstructure which the average grain size of nano TiN was $80 \mathrm{~nm}$, and the surface roughness was $14.6 \mathrm{~nm}$.
\end{abstract}

\section{Introduction}

Nanocoatings are coatings which are formed by components with characteristic dimensionality as nano-size $(1 \sim 100 \mathrm{~nm})$ setting in different matrixes, and own better mechanical performances such like higher hardness, wear resistance and corrosion resistance, etc. [1-3]. Composite electrodeposition technology is a method to obtain composite coatings by adding insoluble micrometer or nanometer sized solid particles (such as TiN, AlN, SiC, and $\mathrm{Al}_{2} \mathrm{O}_{3}$, etc.) into the electrolyte to codeposit the particles and metal matrix with electrodeposition. The electrodeposition of composite coatings can be traced back to studies which produced a graphite-copper composite coating. Due to superiority of both traditional composite materials and modern nano materials, nano composite coatings have become a highlight for researchers all over the world $[4,5]$.

The ultrasonic is a mechanical wave and the frequency is about $2 \times 10^{4} \sim 10^{9} \mathrm{~Hz}$. During nano Ni-TiN composite coatings are prepared, conventional mechanical dispersing method depends on agitation to make the particles suspend in the electrolyte, and is less effective once all particles are suspending, while comparatively the effect of ultrasonic on the dispersion of tiny particles in the electrolyte is more helpful acutely in virtue of its stirring and dispersing effect than mechanical dispersing method can achieve. Artificial neural network (ANN) is a match model that is based on experimental data, and it can get the inner law of the experimental data by a number of iterative computations. In this paper, Ni-TiN nanocoatings were successfully prepared on 45 steel substrates by ultrasonic-electrodeposition. The synthetic conditions of composite coatings were optimized through artificial neural network on the orthogonal test. And the morphologies of the coatings were

\footnotetext{
* Corresponding author. Tel/Fax: +86 459 6507757; E-mail: chunyangandma@163.com
} 
investigated via scanning electron microscope (SEM).

\section{Experimental}

All printed material, including text, illustrations, and charts, must be kept within the parameters of the 8 15/16-inch (53.75 picas) column length and 5 15/16-inch (36 picas) column width. Please do not write or print outside of the column parameters. Margins are 15/16 of an inch on the sides (8 picas), 7/8 of an inch on the top (5.5 picas), and $13 / 16$ of an inch on the bottom (7 picas)

Schematic diagram of the experimental setup used for preparing Ni-TiN composite coatings is shown in Fig.1. Nano Ni-TiN composite coatings were deposited on 45 steel substrates by ultrasonic-electrodeposition process. The anode is a pure Ni plate. The average grain diameters of nano TiN particles (Received from Haitai nano Ltd., PR China) are $20 \mathrm{~nm}$. And the purity is above 99.99\%. The basic compositions of the electrolyte are as follows: $250 \mathrm{~g} / \mathrm{l}$ nickel sulfate, $40 \mathrm{~g} / \mathrm{l}$ nickel chloride, $30 \mathrm{~g} / \mathrm{l}$ boric acid and 2-10 g/l TiN particles. The temperature of the plating bath was kept at $50{ }^{\circ} \mathrm{C}$. The $\mathrm{pH}$ of the plating bath was $4-5$ which adjusted by ammonia water or dilute sulfuric-acid. The current density was $2-5 \mathrm{~A} / \mathrm{dm}^{2}$. Prior to the deposition, the substrates were mechanically polished to a $0.10-0.15 \mu \mathrm{m}$ surface finish, then a sequence of cleanings were performed to remove contamination on the substrate surface, the steel substrates were activated for $15 \mathrm{~s}$ in a mixed acidic bath, then rinsed with distilled water. The Ni-TiN composite coatings were electrodeposited by gradually changing the ultrasonic wave from 0 to $300 \mathrm{~W}$.

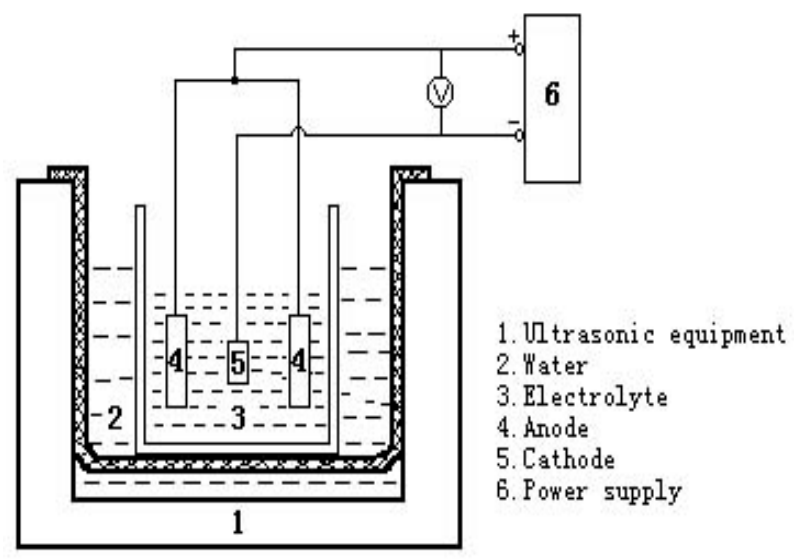

Fig.1. Schematic diagram of the experimental setup

Measured the concentration of Nano-TiN particles in composite coatings with the AA-8600 type of atomic absorption photometry instrument (ASS); the morphologies of the composite coatings were investigated via scanning electron microscope (SEM).

\section{Effects of ultrasonic on nano TiN particles in composite coatings}

Given a certain parameters (the concentration of TiN nanoparticles $7.5 \mathrm{~g} / \mathrm{L}$, the current density 5 $\mathrm{A} / \mathrm{dm}^{2}$, the duty cycle $3: 2$, the concentration of surfactants $80 \mathrm{mg} / \mathrm{L}$ ), the effects of ultrasonic on distribution of nano TiN particles in composite coatings is depicted in Fig. 2. Fig. 2 (a) shows TiN particles in the composite coating which electrodeposited with mechanical stirring instead of the ultrasonic are few in quantity and appear to reunite to some extent. Fig. 2 (b) shows TiN particles in the composite coating which ultrasonic-electrodeposited with appropriate ultrasonic parameters are dispersed homogenously in deposit. Fig. 2 (c) shows TiN particles in the composite coating are rather few in quantity and appear to slight reunite when the coating is prepared by 
ultrasonic-electrodeposited with greater ultrasonic action. The reason is that traditional mechanical dispersing method depends on agitation to make the particles suspend in the electrolyte, and is less effective once all the particles are suspending, while the effect of ultrasonic on the dispersion of tiny particles in the electrolyte is more helpful. Acoustic streams produced by ultrasonic make the TiN particles disperse homogenously in electrolyte, and high-pressure wave and violent stochastic vibration aroused by cavitation effect of ultrasonic shatter the groups of reunited particles, and further homogenize the particles in microscopy. According to the Guglielmi's adsorption mechanism model of composite electrodeposition, the adsorption process of particles on electrode surface may be divided into weak adsorption and strong adsorption, and the weak adsorption process is reversible. When super ultrasonic is adopted, the overmuch cavitation effects of ultrasonic will make the nano TiN particles violent collide and reunite again, while the overmuch stirring effect of ultrasonic will wash the nano TiN particles which infirmly absorb to the cathode surface into the electrolyte again, affect the oriented deposition of the particles, thus decrease the content of nano TiN particles in the composite coating.
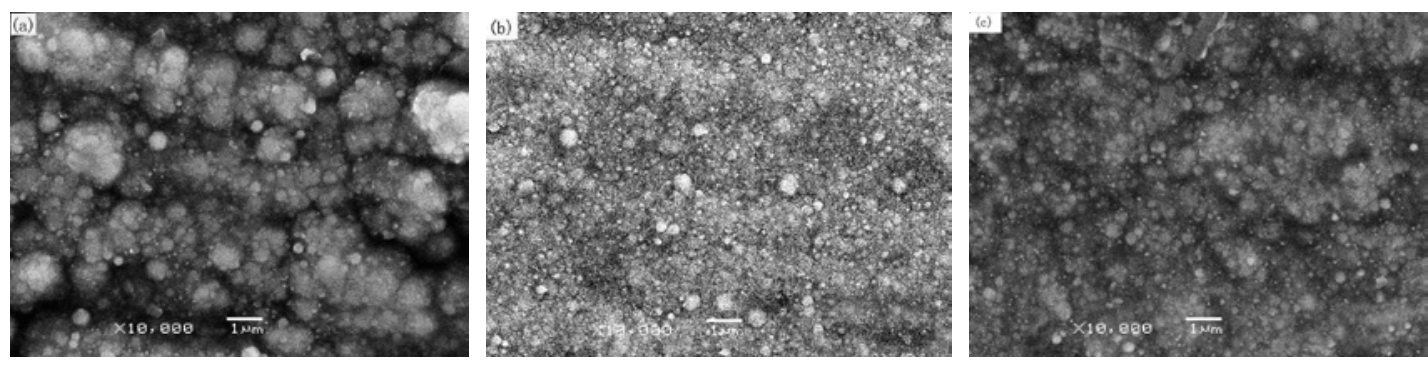

(a) Mechanical stirring, (b) Moderate ultrasonic action (250 W), (c) Super ultrasonic action (300 W)

Fig.2. Distribution of nano TiN particles in composite coating under different action

\section{Forecasted results by ANN model}

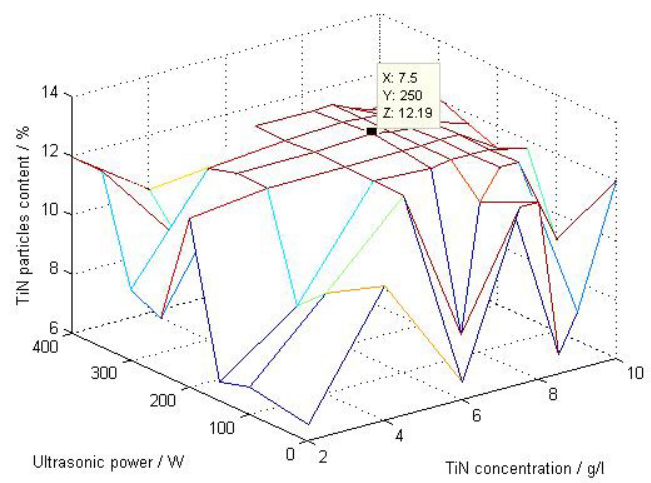

Fig.3. Forecasted result of artificial neural network

Fig. 3 displays the predicted plating parameters of Ni-TiN nanocoatings by using ANN model. It is easily found that the optimal synthetic conditions were determined as follows: the concentration of TiN nanoparticles $7.5 \mathrm{~g} / \mathrm{L}$, the current density $5 \mathrm{~A} / \mathrm{dm}^{2}$, the duty cycle $3: 2$, the concentration of surfactants $80 \mathrm{mg} / \mathrm{L}$ and the ultrasonic power $250 \mathrm{~W}$. It is also revealed that the Ni-TiN nanocoating prepared under the optimal synthetic conditions had a compact and fine microstructure which the average grain size of nano TiN was $80 \mathrm{~nm}$, and the surface roughness was $14.6 \mathrm{~nm}$ from Fig. 2 and Fig. 3. 


\section{Conclusion}

$\mathrm{Ni}$-TiN nanocoatings were successfully synthesized on the surface of 45 steel substrates by using ultrasonic-electrodeposition method. The plating parameters for preparing Ni-TiN nanocoatings were optimized by using an artificial neural network (ANN) basing on the orthogonal test. And the morphologies of the coatings were investigated via scanning electron microscope (SEM). The results indicated that the optimal synthetic conditions were determined as follows: the concentration of TiN nanoparticles $7.5 \mathrm{~g} / \mathrm{L}$, the current density $5 \mathrm{~A} / \mathrm{dm}^{2}$, the duty cycle $3: 2$, the concentration of surfactants $80 \mathrm{mg} / \mathrm{L}$ and the ultrasonic power $250 \mathrm{~W}$. The Ni-TiN nanocoating prepared under the optimal synthetic conditions had a compact and fine microstructure which the average grain size of nano TiN was $80 \mathrm{~nm}$, and the surface roughness was $14.6 \mathrm{~nm}$.

\section{Acknowledgement}

This work has been supported by the Natural Science Foundation of China (Granted no. 51474072) and the Graduate Innovative Research Project of Northeast Petroleum University (YJSCX2016-023NEPU).

\section{References}

[1] C.Y. Ma, G.Q. Liang, Y.Y. Zhu, H.W. Mu, F.F. Xia, Preparation and corrosion assessment of electrodeposited Ni-SiC composite thin films, Ceramics International, Vol. 40(2) (2014), p. 3341.

[2] Baumann S.O., Elser M.J., Auer M., Bernardi J., Diwald O.. Solid-solid interface formation in $\mathrm{TiO}_{2}$ nanoparticle networks. Langmuir, Vol. 27 (2011), p. 1946.

[3] Sen R.J, Bhattacharya S., Das S.H., Das K.B.. Effect of surfactant on the co-electrodeposition of the nano-sized ceria particle in the nickel matrix. Journal of Alloys and Compounds,, Vol. 489 (2010), p. 650.

[4] Heidari G., Tavakoli H., Mousavi Khoie S.M.. Nano SiC-Nickel composite coatings from a sulfamat bath using direct current and pulsed direct current. Journal of Materials Engineering and Performance, Vol. 19(2010), p. 1183.

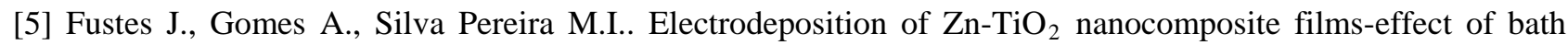
composition. Journal of Solid State Electrochemistry, Vol. 12 (2008), p. 1435. 